\title{
Les Alpes pennines au temps d'Oetzi vers 3200 av. J.-C.
}

\author{
(momie du Hauslabjoch)
}

\author{
A. Bezinge ( $\left.{ }^{1}\right)$ \\ Ingénieur indépendant \\ P. Curdy $\left({ }^{2}\right)$ \\ Musée national suisse
}

\section{Q QUELQUES DONNÉES SUR OETZI}

Le 19 septembre 1991, dans le Oetztal, à proximité du Hauslabjoch (Tyrol du Sud), un cadavre prisonnier des glaces est mis au jour à $3200 \mathrm{~m}$ d'altitude $[1,2]$.

Cette découverte, qui au premier abord n'eut pas paru extraordinaire (on a dans un premier temps cru retrouver le corps d'un alpiniste du $\mathrm{Xx}^{\mathrm{e}}$ siècle), devint rapidement un cas archéologique exceptionnel du fait de l'état de conservation du corps, desséché, momifié et conservé par les glaces, et des objets qu'il avait avec lui. L'homme était vêtu de cuir et de fourrure. Il portait une cape en jonc tressé. Ses chaussures étaient doublées de foin. L'individu transportait avec lui un équipement complet : une hache de cuivre emmanchée (bois d'if), un poignard en silex et une sacoche en herbes tressées; pour la chasse, un arc, un carquois avec 14 flèches dont 2 étaient équipées de pointes en silex. En complément, on a dégagé une armature de portage en noisetier avec des lattes en mélèze ; la momie du Hauslabjoch avait avec elle des provisions de céréales, des prunes sauvages et des morceaux de viande, un nécessaire de pièces à feu avec de l'amadou. Tout ceci nous montre un homme bien équipé pour une expédition dans les Alpes.

Les datations au carbone 14 effectuées par différents laboratoires de recherches ont donné un âge de 4500 ans
(4 $523 \pm 27$ BP et $4576 \pm 27$ BP), soit $3350-3100$ av. J.-C. en datation calibrée avec un écart-type de 2 sigma (degré de certitude de $95 \%$ ), correspondant à la fin de la période climatique de l'Atlantique et en chronologie préhistorique à la fin du Néolitique moyen. Suite à cette découverte, une polémique s'est tout d'abord déclenchée pour nier :

— la validité des dates ${ }^{14} \mathrm{C}$;

- l'existence possible d'un cadavre conservé intact dans les glaces pendant plus de 4000 ans.

Par la suite cette découverte s'est avérée authentique et conforte les hypothèses sur l'occupation ou le passage des hommes au Néolithique en haute altitude.

\section{II $\square$ CLIMAT DANS LES ALPES À L'ÉPO- QUE DE OETZI}

Le milieu du quatrième millénaire BP correspond au passage de la période climatique de l'Atlantique à celle du Subboréal ; la première, en moyenne plus chaude et plus humide que de nos jours, couvre 3000 ans environ, entre 7500 et 4500 BP. Le début du Subboréal voit en gros un climat plus sec et plus frais régner sous nos latitudes.

(') Ingénieur, Chemin des Amandiers 62, 1950 Sion.

(2) Musée national suisse, Hardturmstr. 185, 8005 Zürich.

\section{Pennines Alps at the time of Oetzi (abart 3200 BC)}

The following study describes briefly the climate and its influence on the alpine passes and the glaciers at the time of Oetzi and the state of archeological knowledges at high altitude at the neolithic time. It demonstrates that a number of witness attests the presence of the mankind at high altitude in the Pennines Alpes. The discovery of a prehistoric mummy in Tyrol confirms the process of the colonization of high altitudes or the possible use of the alpin ways at that time. 
On estime que toutes les avancées glaciaires postérieures à $9500 \mathrm{BP}$ n'ont jamais atteint le stade de 1850 ap. J.-C. [3]. Lors des périodes de réchauffement, les glaciers devaient être semblables en volume et en surface à ceux de 1975 et lors de périodes froides, leur état était, à part quelques exceptions, proche de ce qui a été observé en 1850 .

Concernant les cols actuellement englacés tels le Théodule, le Col d'Hérens ou le Col Collon, on peut préciser :

- à la fin d'une période chaude, avec un fort retrait des glaciers, ces cols peuvent être dégagés ; les marges proglaciaires sont alors chargées de matériaux morainiques; l'accès est facilité en été et en automne ;

- lors d'une période froide, les importantes chutes de neige et les faibles fontes estivales facilitent l'accès aux cols en automne car les névés et ponts de neige résiduels sont consolidés.

Vers 1950 , une polémique a été engagée dans la revue du Club Alpin Suisse au sujet de l'influence du climat sur les cols alpins englacés. Certains ont prétendu que des cols fortement englacés étaient impraticables, d'autres au contraire, assuraient que le passage en était plus aisé. Il s'avère qu'un glacier en crue, épais et peu crevassé, peut faciliter l'accès au col, comme cela a été le cas pendant le dernier épisode glaciaire : on a par exemple acheminé les troupeaux par névés et glaciers en direction du Col Collon, entre le Val d'Hérens et le Val d'Aoste. En période de réchauffement, il faut relever que les difficultés d'accès étaient dues surtout aux torrents glaciaires, difficilement franchissables en été. Seul le passage à gué des deltas ou par les fronts glaciaires autorisait le transit estival. Par contre, l'automne, avec la disparition des neiges et les faibles débits torrentiels, devait être une saison privilégiée.

En montagne, lors de périodes chaudes, les glaciers perdent en principe en puissance et les fronts glaciaires reculent. A l'extérieur des moraines, des horizons humifères se mettent en place et une forêt d'altitude, en général formée par des mélèzes, peut se développer sur les marges proglaciaires. Puis, lors du retour d'une péjoration climatique, les glaciers s'avancent dans les vallées, ensevelissent les arbres, et les sols sont recouverts par de nouvelles moraines.

Les datations au ${ }^{14} \mathrm{C}$ effectuées sur des troncs fossiles ou sur les sols précités permettent de suivre sur 8000 à 10000 ans, plusieurs fluctuations climatiques (épisodes de réchauffement ou de refroidissement $[3,4]$ ). Ces dates sont évidemment approximatives de par les incertitudes liées à la méthode de datation (variation de la teneur en ${ }^{14} \mathrm{C}$ atmosphérique au cours du temps, type d'échantillon, etc.). La calibration des dates ${ }^{14} \mathrm{C}$ par les courbes basées sur la dendrochronologie (courbes de calibration) permet de corriger la valeur moyenne de ces dates, avec une précision variable qui dépend du comportement des courbes ellesmême. En réalité, la valeur obtenue en années avant J.-C. n'atteint jamais une précision allant en deçà du siècle pour un degré de certitude de $95 \%$ (écart-type de 2 sigma). Tout phénomène de durée inférieure est donc difficilement individualisable par cette méthode (variation courte, infraséculaire).

L'analyse de la densité des cernes de croissance des arbres permet la mise en place d'une courbe moyenne des températures, domaine de la dendroclimatologie. Le lien entre la courbe dendroclimatique et le climat concerne principalement les variations de la température moyenne estivale qui a une influence sur la densité des cernes du bois ; la température a également une incidence sur les fluctuations glaciaires; ainsi, la dendroclimatologie et la glacio-morphologie sont directement liées et complémentaires.

Partant de ces observations multiples, les diverses études climatologiques ont défini des scénarios globalement concordants : on se situerait vers 4500 BP (3200 av. J.-C.) en principe au début d'un réchauffement, suite à une dégradation climatique (phase de Piora ou Rotmoos), mais avec des fronts glaciaires pas trop éloignés de ce que l'on observe de nos jours $[4,3]$.

Toutefois, il est possible que de courtes périodes fraîches avec neiges permanentes à l'altitude d'Oetzi aient pu se produire de façon sporadique.

\subsection{Les Alpes pennines au Néolithique}

La limite supérieure de la forêt devait se situer à cette période vers $2300 \mathrm{~m}$, mais avec des variations selon les régions (microclimats). Plusieurs colonnes palynologiques faites en altitude dévoilent certains faits particuliers : présence à l'étage subalpin supérieur de niveaux d'incendie (brûlis) ou remplacement de certaines espèces de résineux par l'aulne vert - phénomènes que l'on a rattachés à une présence humaine sporadique [5,6]. Les Néolithiques auraient donc pu à cette époque aménager et utiliser les prairies d'altitude pour l'estivage des troupeaux (chèvres et moutons principalement). Ces faits qui demandent à être étayés, paraissent du moins ne pas être systématiques et ne pas entraîner une transformation irréversible du milieu comme ce sera le cas aux époques postérieures.

\subsection{La vallée du Rhône au Néolithique}

Les découvertes archéologiques recensées en Valais révèlent la présence de l'homme au niveau de la plaine dès la période mésolithique, entre 8 000-7 500 BP (7000-6200 av. J.-C., [7]). Les cols alpins, englacés ou pas, ont dû certainement être abordés par des groupes de chasseurscueilleurs (territoires de chasse estivale, sites d'exploitation de matière première, du cristal de roche en particulier), mais les preuves archéologiques manquent encore en $\mathrm{Va}$ lais. Cette colonisation est attestée ailleurs dans le TrentinHaut Adige, où des sites d'altitude datés du Mésolithique ont été établis vers $2000 \mathrm{~m}$ [8]. Plus récemment, on a découvert à l'Alpe Veglia, à près de $2000 \mathrm{~m}$, non loin du col du Simplon, un gisement de plein air daté sous réserve de cette époque [9].

Le Néolithique (environ 5000-2500 av. J.-C. en Suisse) voit le premier développement de communautés de pasteurs-agriculteurs dans les Alpes (en dernier lieu [10]) ; en haute vallée du Rhône, les habitats sont situés audessous de $1000 \mathrm{~m}$ d'altitude, au niveau de la plaine, ou près du débouché de quelques grandes vallées latérales (Val d'Hérens ou Val de Bagnes par exemple).

Entre 4000 et 3300 av. J.-C. (Néolithique moyen 2 valaisan), le peuplement de la vallée du Rhône est bien stabilisé en plaine. La période suivante, 3300-3000 av. J.-C., dénote un hiatus dans les dates ${ }^{14} \mathrm{C}$ disponibles en Valais : difficile à interpréter, il pourrait soit correspondre à un déplacement probable des sites (qui restent donc à découvrir), soit plus vraisemblablement à un hasard de 
l'échantillonnage des dates ${ }^{14} \mathrm{C}$ disponibles [10]. Le Néolithique final (3000-2500 av. J.-C.) est également représenté en plaine.

Il semble bien que jusqu'à la fin du Néolithique, l'étage montagnard (entre environ 1000 et $1400 \mathrm{~m}$ ) n'ait pas encore été utilisé de manière systématique par les communautés agro-pastorales; cette montée en altitude de l'habitat (sites permanents) n'aurait lieu qu'à partir de l'âge du Bronze vers le début du $2^{\mathrm{c}}$ millénaire avant J.-C.

Pourtant quelques trouvailles néolithiques d'altitude (objets isolés) jalonnent les chemins qui conduisent des vallées latérales aux cols des Alpes pennines et aux zones de pâturage limitrophes. La plupart sont malheureusement mal datées $\left(5^{\mathrm{c}}-3^{\mathrm{e}}\right.$ millénaire, av. J.-C. $)$.

\subsection{Liste des trouvailles et sites d'altitude}

Col de l'Albrun, vallée de Binn (fig. $l \mathrm{n}^{\circ} 1$ ) relie la vallée de Conches au Val d'Ossola. Une hache en serpentinite a été mise au jour sur le chemin menant au col [11]. Selon cet auteur, de tous les cols du Haut-Valais, l'Albrun serait celui qui permettait l'accès le plus aisé au sud des Alpes.

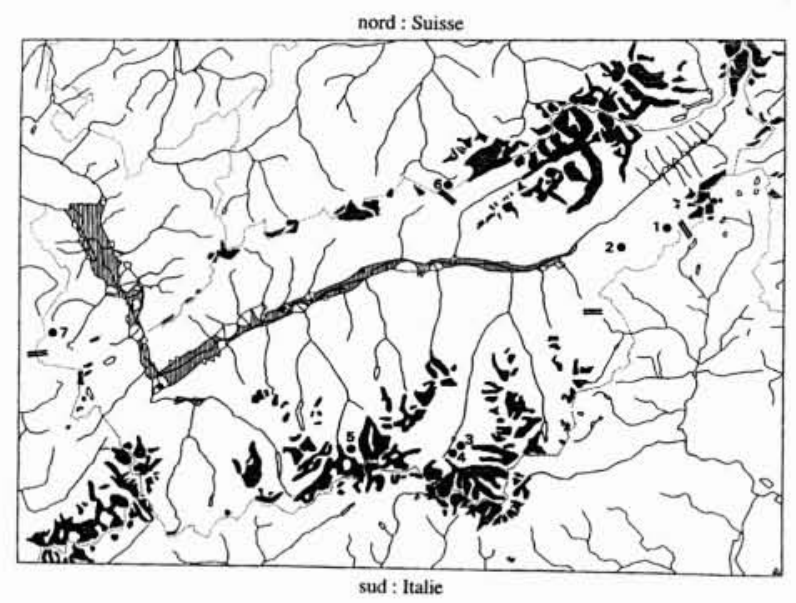

1. Carte de distribution des vestiges néolithiques en altitude (Alpes du Valais et vallée du Rhône).

A Grengiols, vallée de Conches (fig. $l \mathrm{n}^{\circ} 2$ ), une pointe en silex du Grand Pressigny (Néolithique final) a été récoltée au-dessus de Grengiols dans la forêt ou peut-être plus haut à près de $2500 \mathrm{~m}$ d'altitude $[12,13]$.

Zermatt, Furgg ( 2400 m, fig. $1, \mathrm{n}^{\circ} 3$ et fig. 2$)$ : en 1959 , lors du percement d'un tunnel effectué dans le cadre des travaux d'aménagements de la Grande Dixence, on a récolté une hache en éclogite à $2400 \mathrm{~m}$ d'altitude près du chemin menant au col du Théodule.

La forme de cette pièce assez particulière ne se rencontre que rarement dans les haches néolithiques du Valais ; elle évoquerait plutôt la zone atlantique et plus précisément la Bretagne, où elle est datée du $5^{\mathrm{c}}$ millénaire avant $\mathrm{J}$.-C. (en dernier lieu [14]). La détermination pétrographique indique qu'il s'agit d'une éclogite ; or à Zermatt les gisements affleurent non loin du lieu de découverte ; la disposition des minéraux permettrait selon certains de trancher pour

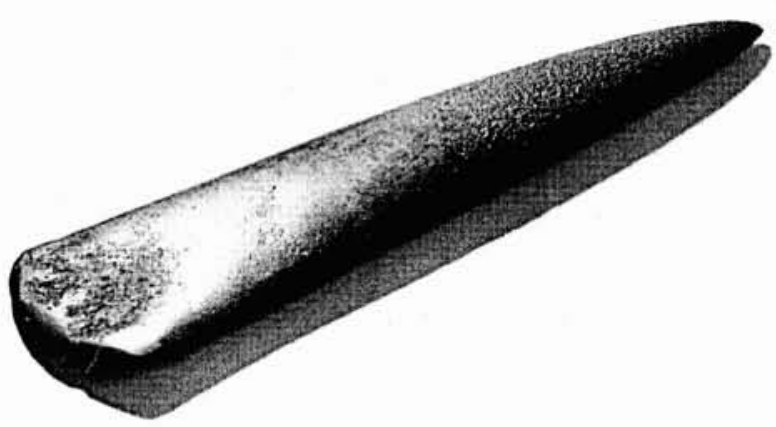

2. Hache néolithique en éclogite découverte au-dessus de Zermatt (Photo H. Preisig, Sion).

une importation en provenance de la région de Nantes. Une analyse pétrographique devrait être reconduite, en tenant compte des caractéristiques des affleurements locaux de la région zermattoise et l'hypothèse d'une production locale ne peut pas être écartée.

La présence d'une pièce aussi exceptionnelle non loin du passage du Théodule pose problème: elle pourrait témoigner de l'utilisation du col pour les échanges entre la vallée de Zermatt (ou le Val d'Hérens) et le Valtournenche ; a-t-elle été accidentellement perdue par son utilisateur, un berger ou prospecteur de pierre verte/de cristal de roche ? ou encore, de par la valeur de prestige que l'on serait enclin à porter à une pièce aussi rare, s'agit-il d'un dépôt volontaire, rituel lié au passage du col ? Ce phénomène d'offrandes votives - faites entre autres pour s'assurer les faveurs des divinités du lieu et se protéger des dangers d'un parcours difficile - est par ailleurs bien marqué à d'autres époques : dépôts d'altitude de l'âge du Bronze [15], sanctuaire gallo-romain du Grand-Saint Bernard [16], etc.

Zermatt, abri de pied de paroi à Alp Hermettji (2 $560 \mathrm{~m}$, fig. $1, \mathrm{n}^{\circ} 4$ ): en 1985 , à l'occasion d'une campagne de prospection archéologique dans la vallée de Zermatt, le groupe PAVAC (Université de Genève) met au jour une séquence d'occupations néolithiques dans un petit abri au pied d'une paroi à $2580 \mathrm{~m}$ d'altitude [17]. Ce site fait actuellement l'objet d'investigations plus approfondies (fouilles du Musée national en 1993). Les datations au ${ }^{14} \mathrm{C}$ situent les plus anciennes occupations au début du $5^{\mathrm{e}}$ millénaire avant notre ère (charbons provenant d'un foyer, datés de $5740 \pm 40$, soit $4760-4500$ av. J.-C.). Une couche plus récente a été datée du Néolithique final $(4330 \pm 40$, 3080-2890 av. J.-C.). Entre ces dates extrêmes, les niveaux archéologiques démontrent une occupation sporadique saisonnière des lieux. Cet abri est situé au-dessus d'une zone favorable à la pâture estivale mais également à l'emplacement d'affleurements de roche verte et en particulier d'éclogite. On pourrait donc lier l'utilisation de ce couvert à l'exploitation du terroir pastoral en été ou à l'extraction de roche verte. Les quelques outils mis au jour, pointes de flèche, vase en céramique ou éclats de cristal de roche ne renseignent guère sur la fonction du site. Autre hypothèse, il pourrait s'agir d'une halte-refuge sur la voie de passage entre le Val d'Aoste et le Valais central, via les cols d'Hérens et du Théodule, dont la fréquentation est au moins attestée à l'époque historique [18].

Pointe de silex du Grand Pressigny au Plan Bertol ( $2600 \mathrm{~m}$, fig. $\left.I \mathrm{n}^{\circ} 5\right)$ : en 1948, on a trouvé au Plan Bertol, 
au-dessus d'Arolla, une pointe en silex jaune du Grand Pressigny, datée du Néolithique final [12]. Le Plan Bertol est directement sur la voie du col Collon, qui conduit du Val d'Hérens au Val d'Aoste, pour celui qui voulait contourner la zone de confluence entre le Haut glacier d'Arolla et le glacier du Mont Collon.

\subsection{Trouvailles néolithiques d'altitude dans les régions limitrophes}

Arcs près du Lötschenpass (fig. $l \mathrm{n}^{\circ} 6$ ) : parmi les divers objets récoltés par le peintre Albert Nyfeler au Lötschental, on connaissait, outre des monnaies et d'autres pièces antiques récoltées sur le versant bernois du col du Lötschenpass (Lötschegletscher), trois arcs en bois ; ces derniers ont été tout récemment datés au ${ }^{14} \mathrm{C}: 3535 \pm 70$, $3795 \pm 55$ et $3555 \pm 65$, soit le Néolithique final ou le début de l'âge du Bronze (2400-1700 av. J.-C.), pour l'ensemble des trois pièces [19]. L'emplacement exact des découvertes n'a pas pu être localisé, mais correspond à l'ancien tracé englacé du passage menant au col (entre 2400 et $2700 \mathrm{~m}$ d'altitude).

Hache en pierre verte de l'alpage de la Pierre sur Champéry $\left(1600 \mathrm{~m}\right.$, fig. $\left.1 \mathrm{n}^{\circ} 7\right)$ : cette pièce a été mise au jour fortuitement par un promeneur en 1988 [20]. Là encore, tout en faisant observer la présence à proximité d'un col (col de Coux, $1924 \mathrm{~m}$ ), on tendrait pourtant à mettre en relation la découverte de cet outil avec l'utilisation des pâturages environnants.

Grisons et Suisse centrale : en dehors de la zone pennine, outre les sites de la région Trentin-Haut Adige déjà mentionnés, et quelques trouvailles isolées probablement néolithiques dans les Grisons [21], on peut citer les recherches récentes effectuées en Suisse centrale, une région particulièrement pauvre en vestiges d'époque préhistorique ; on y a par exemple repéré un atelier de débitage de cristal de roche daté de la fin du Néolithique/début de l'âge du Bronze à plus de $2000 \mathrm{~m}$ d'altitude [22].

\section{CONCLUSIONS}

Il est assuré qu'au Néolithique, à la fin de l'Atlantique/début du Subboréal, l'homme a pénétré les zones de haute altitude en Valais comme dans les autres régions alpines, et ceci pour plusieurs raisons.

- L'utilisation des prairies (alpages) pour le bétail en transhumance estivale, bien que la preuve directe de cette pratique fasse défaut; les palynologues ont rattaché les traces de "défrichements" de la fin du Néolithique, en limite supérieure de la forêt à l'extension des zones de pâtures. Ce sujet demanderait à être approfondi ; il est par contre certain au vu des données disponibles, que cette pratique ne soit intervenue qu'au cours du Néolithique final, et de ce fait à une période d'amélioration climatique marquée, au début du Subboréal. Mais le critère climatique n'est en soi pas nécessairement plus significatif que d'autres - pression démographique, innovation dans le système pastoral, etc.

- L'exploitation de matière première : pierre verte, cristal de roche.

- L'utilisation des passages d'altitude dans les échanges : échanges de proximité, de vallée à vallée, dans le cadre restreint de communautés montagnardes utilisant un même espace bio-géographique ; dans ce cas, tous les cols, englacés ou pas, ont pu être utilisés.

Il est évident que la situation climatique de l'époque d'Oetzi ou peu après avec des glaciers en retrait devait être favorable à l'utilisation de vastes territoires d'altitude pour l'estivage du bétail ; les communications de vallée à vallée par le haut ne devaient en aucun cas poser des problèmes insolubles à des gens habitués à ces régions. $\mathrm{Si}$ un refroidissement climatique du genre de ce qui a été observé au Moyen Age avec le Petit Age glaciaire a pu entraîner certaines difficultés dans l'utilisation de ces passages, voire même la fermeture temporaire de certains d'entre eux, on ne doit pas pour autant en déduire l'abandon rapide et systématique d'un système agro-pastoral qui a dû utiliser les hautes altitudes pour l'estivage du bétail.

Ces passages d'altitudes étaient même à la base de liens privilégiés entre vallées de part et d'autre de la chaîne alpine. Des affinités culturelles marquées sont d'ailleurs attestées au cours de toute la Préhistoire; la première colonisation néolithique semble bien avoir pénétré la haute vallée du Rhône depuis le Sud [14]; le rite d'ensevelissement en tombe en dalle avec position fœtale du défunt (cistes de type Chamblandes) caractérise également en Valais un faciès d'affinité sud-alpine ; les sépultures collectives du Néolithique final de Sion «Petit-Chasseur » et Aoste «Saint-Martin de Corléans » sont en tous points semblables, le style des gravures des stèles funéraires procèdent d'une même "école » (ibid.). A l'âge du Fer, on voit les caractéristiques de la culture matérielle du Haut Valais très proches de celles du Val d'Ossola et du Tessin [23] ; à la même époque, le Bas Valais présente de fortes affinités avec le Val d'Aoste bien plus qu'avec le Plateau suisse, la région en aval de Massongex/Saint-Maurice étant même définie comme zone frontière entre ce dernier et le monde alpin valaison [24].

En ce qui concerne la rétention du corps d'Oetzi dans les glaciers, il a été nécessaire que le climat soit devenu frais et riche en neige. Les dernières études détaillées sur les variations climatiques démontrent que de courtes périodes fraîches (quelques décennies) se sont produites de façon abrupte avec un abaissement de température d'environ $5^{\circ} \mathrm{C}$.

Il est probable que le corps reposait à sa mort, à même le sol dans le lit rocheux d'une dépression en forme de cuvette. Les neiges l'ont recouvert et se sont transformées en glace. Son corps a pu rester en place dans ce substrat sans mouvement si ce n'est la fonte superficielle. Sur une crête rocheuse, une cuvette représente une rétention privilégiée de la neige qui forme une masse glaciaire immobile.

Un corps emprisonné pendant des milliers d'années peut très bien réapparaître après de longues périodes de sécheresse et d'étés chauds car une déglaciation généralisée est observée dans les Alpes depuis 1940 : tous les glaciers sont en décrue.

Le blocage du corps d'Oetzi est un cas très différent de celui d'un homme tombé dans une crevasse, donc dans des glaces en mouvement, avec une progression moyenne de 10-20 cm par jour ; après plusieurs décennies il réapparaît généralement déformé, "étiré » voire complètement disloqué. Ainsi, les ossements et l'équipement du mercenaire du $\mathrm{XVI}^{\mathrm{C}}$ siècle, découverts entre 1985 et 1990 en bordure 
du glacier du Théodule, à près de $3000 \mathrm{~m}$ d'altitude, étaient dispersés sur plusieurs dizaines de mètres (en dernier lieu, [25]).

En ce qui concerne Oetzi ou Hibernatus, on peut donc affirmer qu'il n'était pas seul à cette altitude dans les Alpes et sa présence n'a rien d'incongru.

Au cours des millénaires, de nombreux «Oetzi » ou « Hibernatus » ont parcouru les Alpes, beaucoup ont perdu la vie et ont disparu corps et biens, sous une avalanche, dans une crevasse, ou par noyade dans un torrent glaciaire. La trouvaille du Hauslabjoch n'est donc qu'une preuve supplémentaire, mais de qualité exceptionnelle, qui confirme la présence de l'homme dans les Alpes aux limites de l'oekoumène.

Etude présentée lors de la $173^{\mathrm{e}}$ assemblée annuelle de la Société suisse des Sciences naturelles à Verbier les 22-26 septembre 1993, dans le cadre des 100 ans de la Commission suisse des glaciers.

\section{Bibliographie}

[1] Höpfel F., Platzer W. et Spindler K. (dir.) (1992). - Der Mann im Eis, Band 1. Bericht über das Internationale Symposium 1992 in Innsbruck.

[2] EgG M. et al. (1993). - GOEDECKER-CiOleK R., Groenman-Van WaAteringe W. et Spindler K. - Die Gletschermumie. Vom Ende der Steinzeit aus den Ötztaler Alpen. Sonderdr. aus Jb RGK 39, 1992. Mainz 1993.

[3] Röthlisberger F, et al. (1980). - Holocene Climatic Fluctuations-Radiocarbon Dating of Fossil Soils (fAh) and Woods from Moraines and Glaciers in the Alps. Geography in Switzerland, 24th internat. Congress. Tokyo, pp. 21 ss.

[4] RENNER F. (1982), - Beiträge zur Gletschergeschichte des Gotthardgebietes und dendroclimatologische Analysen an fossilen Hölzern. Physische Geographie 8, pp. 1 ss.

[5] MARKGRAF V. (1969). - Moorkundliche und vegetationgeschichtliche Untersuchungen an einem Moorsee an des Waldgrenze im Wallis. Bot. Jahrbücher für Systematik, Pflanzengesch. und Pflanzengeografie, 89, pp. 1 ss.

[6] WELTEN M. (1982), - Vegetationgeschichtliche Untersuchungen in den westlichen Schweizer Alpen: Bern-Wallis. Mém. Soc. helvétique des sciences nat., 95.

[7] Crotri P. (1993). - L'épipaléolithique et le Mésolithique en Suisse: les derniers chasseurs. La Suisse du Paléolithique à l'aube du Moyen-Age. SPM I, pp. 205 ss.

[8] Bagolini B. et PEdrotti A.-L. (1992). - Vorgeschichtliche Höhenfunde im Trentino-Südtyrol und im Dolomitenraum vom Spätpaläolithikum bis zu den Anfängen der Metallurgie. In F. Höpfel, W. Platzer et K. Spindler (dir.), Der Mann im Eis, Band 1. Bericht über das Internationale Symposium 1992 in Insbruck, pp. 359 ss.

[9] Guerreschi A., Ghiretti A. et Gambari F.M. (1992). Archeologia all'Alpe Veglia. In : Le Rive 4/5, pp. 33 ss.

[10] BAUdAIS D. et al. (1989/90). - Le Néolithique de la région de Sion. Bull. du Centre genevois d'Anthropologie 2 , pp. $5 \mathrm{ss}$.

[11] Graeser G. (1986). - La vallée de Conches de la préhistoire à l'époque romaine. In : Le Valais avant l'Histoire, Catalogue de l'exposition. Musées cantonaux, p. 302.

[12] SAUTER M.-R. (1950). - Préhistoire du Valais des origines à l'époque mérovingienne. Vallesia V.
[13] PITTARD E. (1920/22). - Sur une pointe de lance néolithique trouvée dans les Alpes suisses à $2500 \mathrm{~m}$ d'altitude. Arch. suisses d'anthropologie générale, IV, pp. 151 ss.

[14] GALLAY A. (1986). - Une hache néolithique bretonne sur le chemin du Col du Théodule. In : Le Valais avant I'Histoire, Catalogue de l'exposition. Musées cantonaux, pp. $86 \mathrm{ss}$.

[15] WiBLÉ F. (1986), - Le Grand Saint-Bernard (Summus Poeninus). In: Le Valais avant I'Histoire, Catalogue de l'exposition. Musées cantonaux, 1986, pp. 216 ss.

[16] WYSS R. (1971). - Die Eroberung der Alpen durch den Bronzezeitmenchen. Zeitschr. Archäologie und Kunstgesch. 28 , pp. $130 \mathrm{ss}$.

[17] MAY O. (1987), - (PAVAC), Zermatt (VS), abri d'Alp Hermettji : un site néolithique à $2600 \mathrm{~m}$ d'altitude. Ann. Soc. Suisse de Préhistoire et d'Archéologie, 70, pp. 173 ss.

[18] RÖTHLISBERGER F. (1976), - 8000 Jahre Walliser Gletschergeschichte, II Teil, Gletscher- und Klimaschwankungen im Raum Zermatt Ferpècle und Arolla. SAC Verlag. Bern, p. 134.

[19] Bellwald W. (1992). - Dreispätneolithisch /frühbronzezeitliche Pfeilbogen aus dem Gletschereis am Lötschenpass. Archéologie Suisse 15.1992.4, pp. 166 ss.

[20] Curdy Ph. (1992). - ChAMPÊRY. In : Wiblé F. (dir.) chronique archéologique dans le canton du Valais en 1991. Vallesia XLVII, p. 322.

[21] CURDY Ph. et al. (1993). - Brig-Glis/Waldmatte, un site d'habitat de l'âge du Fer en milieu alpin. Archéologie suisse 4.1993.4, pp. 138 ss.

[22] Primas M. et al. (1992). - SChmid-Sikimic B. et Della CASA P. - Archäologie zwichen Vierwaldstättersee ung Gotthard. Universitätsforschungen zur prähist. Archäologie. 2. Zurich 1992.

[23] MEYER W. (1992). - Die Söldner vom Theodulpass und andere Gletscherfunde aus der Schweiz. In : F. Höpfel, W. Platzer et K. Spindler (dir.), Der Mann im Eis, Band 1, Bericht über das Internationale Symposium 1992 in Inssbruck, pp. $321 \mathrm{ss.}$

[24] Haldimann M.-A. et al. (1991). - Curdy Ph., Gillioz P.-A., KAEnel G. et WiBlé F. - Aux origines de Massongex VS. Tarnaiae, de La Tène finale à l'époque augustéenne. Ann. Soc. suisse de Préhistoire et d'Archéologie 74, pp. 129 ss.

[25] Baudais et al. (1987). - Baudais D., Curdy Ph., DavidElBILI M., MAY O. - Prospection archéologique du Valais. Une approche du peuplement préhistorique. Archéologie suisse 10.1987.1, pp. 2 ss.

[26] Bezinge A., ViviAn R. (1976). - Bilan de la section de glaciologie de la société hydrotechnique de France: sites sous-glaciaires et climat de la période holocène en Europe. La Houille Blanche, $\mathrm{n}^{\circ} 6 / 7$, pp. 00.

[27] David-Elbiali M. (1990). - L'âge du Bronze en Valais et dans le Chablais vaudois. Ann. Soc. Suisse de Préhistoire et d'Archéologie 73,., pp. 19 ss.

[28] SAUTER M.-R. (1978), - Une hache bretonne néolithique sur le chemin du Théodule (Zermatt, Valais). Vallesia, 33, pp. 1 ss.

[29] ZÜRCHER A. (1982). - Urgeschichtliche Fundstellen Graubündens. Chur. 